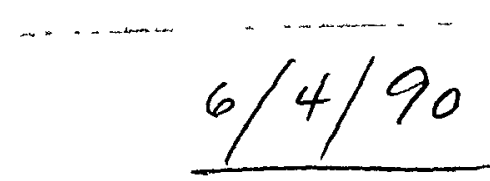

\title{
COUPLED THERMAL AND ELECTRICAL ANALYSIS OF OBSTRUCTED RTGS
}

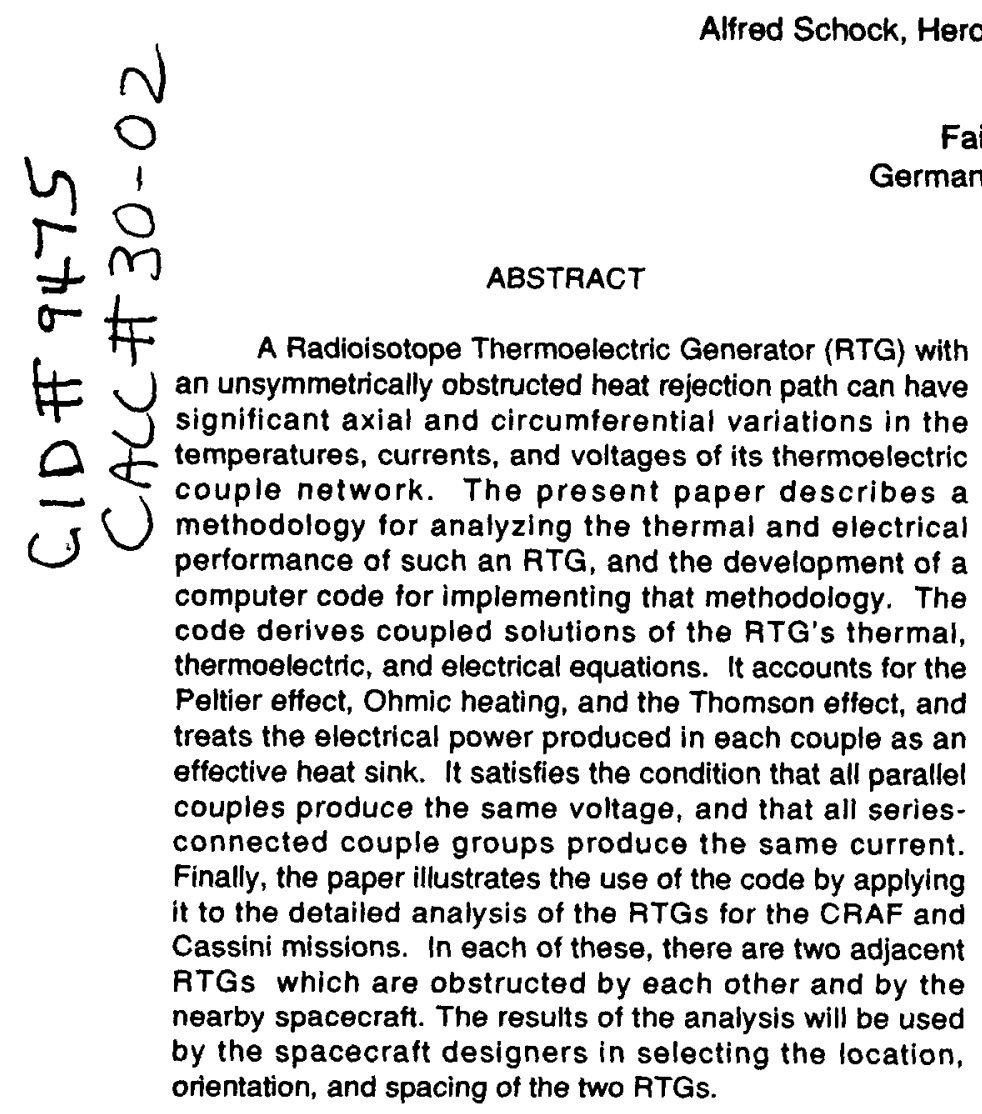

\section{INTRODUCTION}

Previous RTG analyses usually made the simplifying assumption that all of the thermoelectric couples in the generator's series-parallel network operate at the same hotand cold-junction temperatures and at the same current and voltage. For unobstructed RTGs, such a simplified analysis is a useful initial design tool, since it permits closed-form solutions for the optimum area ratio $A_{n} / A_{p}$ of the thermoelectric $n$ - and $p$-legs and for the optimum output voltage. For these optimized parameters, it yields simple expressions for the maximum material efficiency of the thermoelectric couples, and for the required RTG design parameters [1,2].

The above simplified models are not really valid even for RTGs with an unobstructed view of space, because they have significant axial temperature variations due to unavoidable end losses by radiation and by conduction through the heat source support structure. A more exact analysis, which accounted for the axial temperature variations in a Martian RTG, was reported last year $[3,4]$. But even that analysis assumed that the RTG has an axisymmetric view of space and the Martian ground, and therefore no circumferential temperature variation.

The present paper develops a Fairchild-generated methodology and generalized computer code for analyzing the performance of arbitrarily obstructed RTGs with both axial and circumferential temperature, voltage, and current variations, and applies that methodology to the specific example of obstructed RTGs for the upcoming Comet Rendezvous and Asteroid Flyby (CRAF) mission and for the Cassini mission. Both of these are part of the MarinerMark-ll series of missions, which will use a new generation of cost-effective modular spacecraft that can easily be modifled to accomplish a variety of missions to comets and asteroids and to the outer solar system. CRAF, which is scheduled for a 1995 launch, will fly by at least one asterold, orbit a comet, and launch an instrumented penetrator/lander into the comet's nucleus. Cassini, scheduled for a 1996 launch, will also fly by at least one asteroid, fly by Jupiter, orbit the planet Saturn, repeatedly fly near many of Saturn's moons, and send an instrumented probe, called the Huygens probe, into the atmosphere of Saturn's moon Titan. Both spacecraft will employ the same GPHS-RTG design $[5,6]$ used on the recently launched Galileo mission and on the Ulysses mission to be launched in October 1990. In both the CRAF and Cassini missions, the spacecraft designers wish to know the effect of locating two RTGs in close proximity and at odd angles to each other, and also to the nearby spacecraft. The resultant mutual obstruction can result in significant axial and circumferential variation of the RTGs' cold-junction temperatures and the couples' electrical pertormances. The results of the analysis will guide the spacecraft designer in deciding how close the two RTGs can be located near each other and near the spacecraft without experiencing excessive performance penalties. 


\section{DISCLAIMER}

This report was prepared as an account of work sponsored by an agency of the United States Government. Neither the United States Government nor any agency Thereof, nor any of their employees, makes any warranty, express or implied, or assumes any legal liability or responsibility for the accuracy, completeness, or usefulness of any information, apparatus, product, or process disclosed, or represents that its use would not infringe privately owned rights. Reference herein to any specific commercial product, process, or service by trade name, trademark, manufacturer, or otherwise does not necessarily constitute or imply its endorsement, recommendation, or favoring by the United States Government or any agency thereof. The views and opinions of authors expressed herein do not necessarily state or reflect those of the United States Government or any agency thereof. 


\section{DISCLAIMER}

Portions of this document may be illegible in electronic image products. Images are produced from the best available original document. 
ANALYSIS

To analyze an RTG of a given design, orientation, and obstructed heat rejection path, a detalled threedimensional thermal model of the RTG and its environment must be constructed. The hot junction and cold junction of each thermoelectric element in the RTG are represented as discrete nodes. The model cannot be analyzed by means of a standard thermal analysis code, because the connectors between the couple's hot and cold Junctions are not simple thermal conductors. In addition to normal heat conduction, the Peltier effect, Onmic dissipation, and Thomson effect also affect the rate at which heat enters the connector's hot end and leaves its cold end. Moreover, these two rates are not equal, since part of the heat entering each couple is converted to electrical energy. That part must be represented as an effective heat sink for each couple.

As will be shown, the magnitude of the Peltier, Ohmic, and Thomson effects In each couple are functions of the couple current I, which therefore affects each couple's effective thermal conductance and heat sink. Consequently, the RTG's thermal analysis cannot be performed separately, but must be done simultaneously with its thermoelectric and electrical analyses. The electrical analysis is complicated by the constraint that the RTG's thermoelectric couples are in general interconnected in a complex series-parallel network, and that all couples grouped in parallel must operate at the same output voltage, and that all couple groups in series must produce the same current.

Figure 1 shows a cutaway view of the GPHS-RTG that will be used on the CRAF and Cassini missions. Each RTG contains a total of 576 thermoelectric unicouples, arranged in a cylindrical array of 36 layers consisting of 16 couples per layer. As shown in Figure 2, each unicouple contalns a thermoelectric $n$ - and $p$-leg. They are electrically connected at their hot ends by a hot shoe, which serves to collect the heat radiated by the centrally located heat source stack and deliver It to the thermoelectric legs. The cold end of each leg is series- and parallel-connected to adjoining couples to form the RTG's electrical network. The couples' cold ends are bolted to the RTG housing, to which they reject their waste heat. The RTG's waste heat is dissipated by radiation from its housing and its eight equispaced radiator fins, as shown in Figure 1.
Figure 1. GPHS RTG

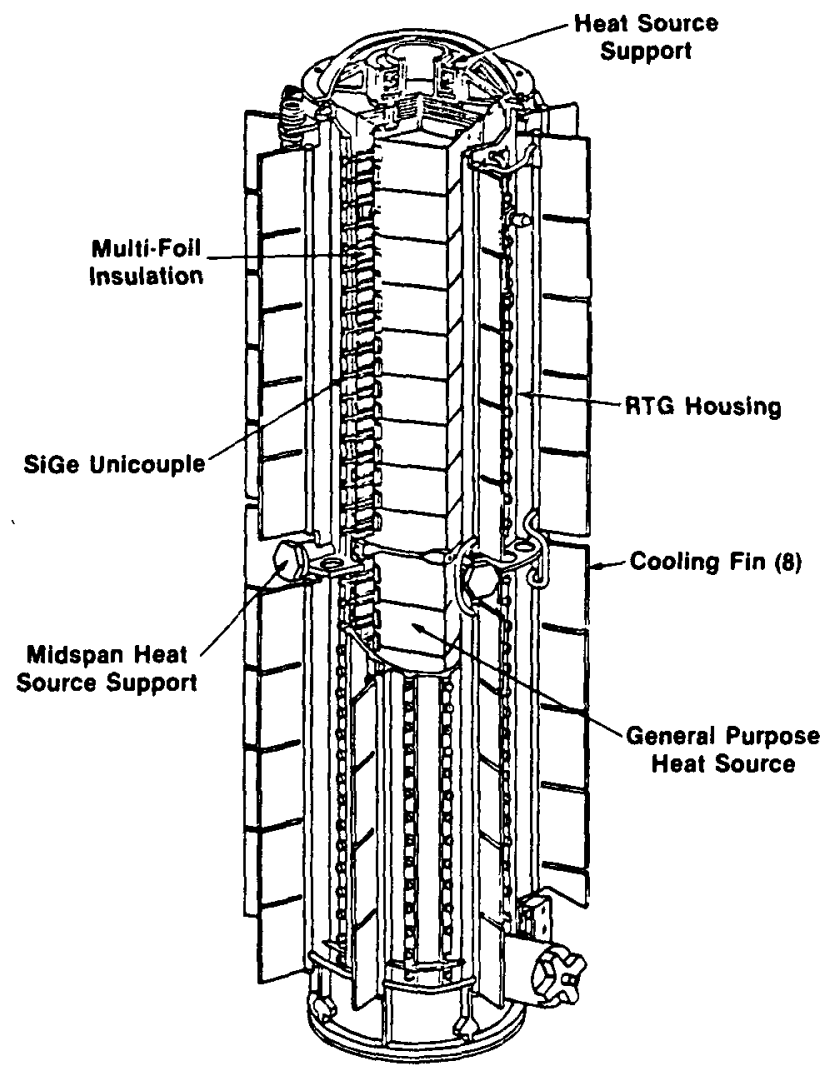

Figure 2. Thermoelectric Unicouple

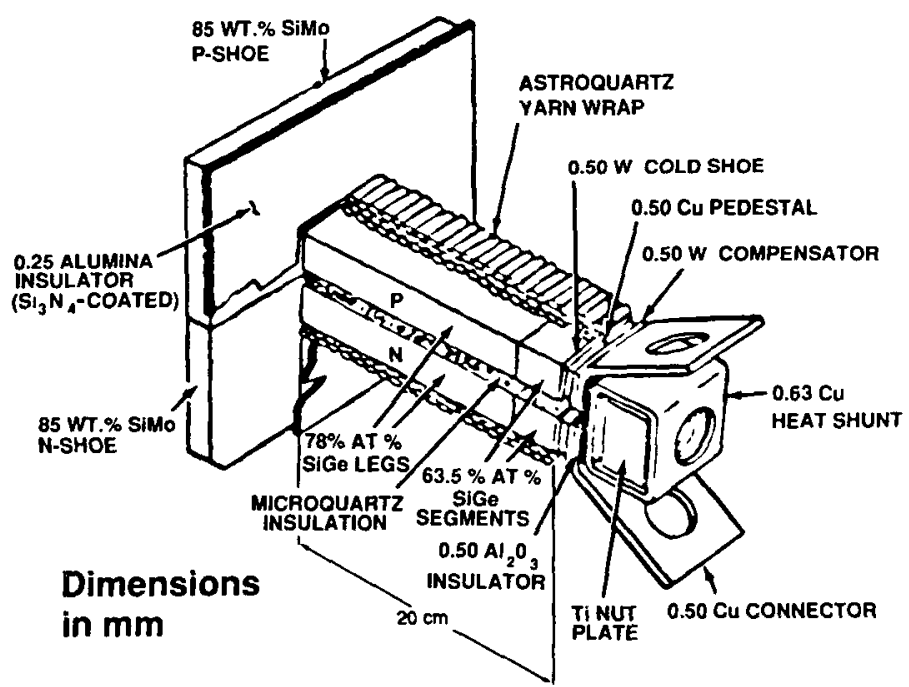




\section{RTG Themoelectric Couple Network}

The series-parallel network of the GPHS-RTG is shown in Figure 3, which depicts a rolled-out schematic of the cylindrical array. The electrical network consists of two parallel branches. Each branch consists of 144 seriesconnected groups of two parallel couples. The rather tortuous current path shown is designed to minimize the ATG's self-induced magnetic field.

\section{Figure 3. Current Path Through GPHS RTG}

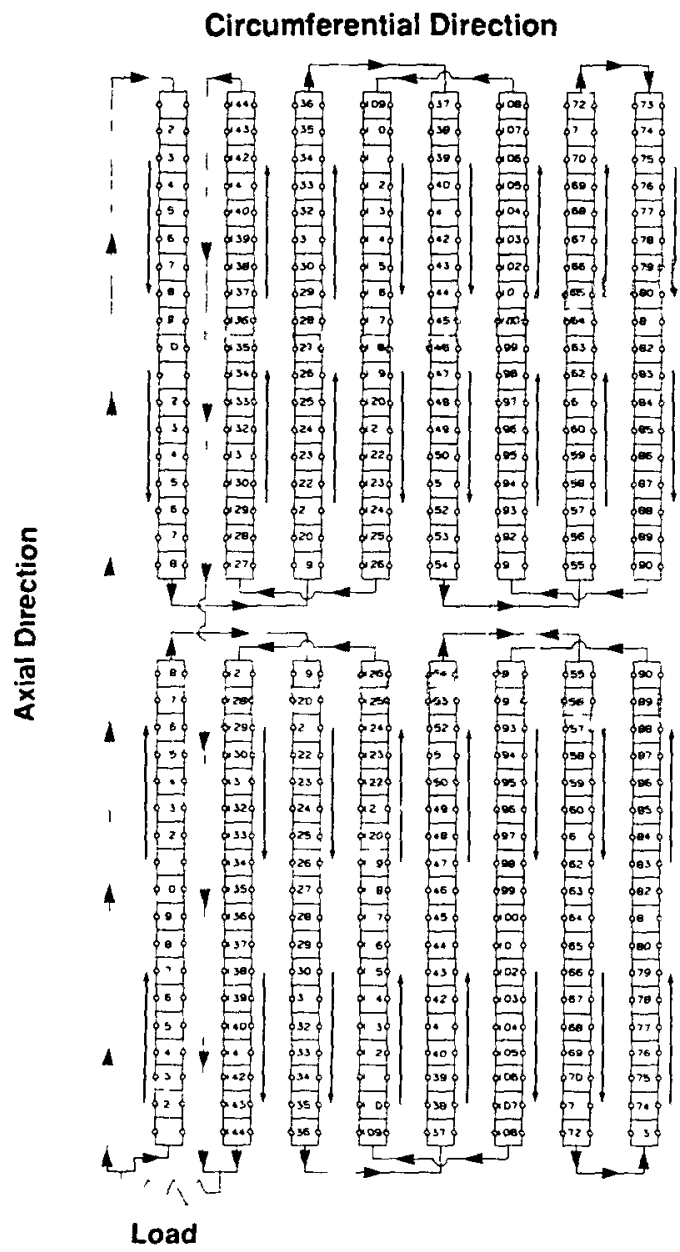

Branch I

The Fairchild RTG analysis methodology is generic, not just for the GPHS-RTG. In general, the equivalent circuit of an RTG network consists of B parallel branches, with each branch containing $G$ series-connected groups of C parallel couples. Each thermoelectric element in the ATG is designated by a branch number $b$, group number $g$ and couple number c, where:

$$
\begin{aligned}
& l<b \leq B, \\
& l<8 \leq G, \\
& l<c \leq C
\end{aligned}
$$

Branch 2
For the case of the GPHS (Gallieo-class) ATG, $B=2$, $G=144, C=2$, and the equivalent circuit of the generator is shown in Figure 4.

Figure 4. Equivalent Circuit of GPHS RTG

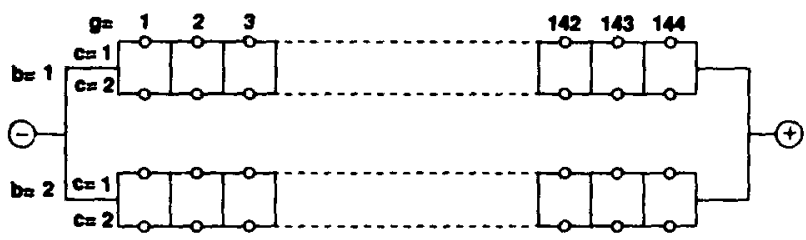

\section{Computing the Couple Conductances and Resistances}

- The code starts with:

- The temperature-dependent thermal conductivity $k$, electrical resistivity $\rho$ and Seebeck coefficient $S$ of the thermoelectric $n$ - and $p$-materials.

- The length $L$ and cross-sectional areas $A_{n}$ and $A_{p}$ of the TE legs, and

- The cold- and hot-junction temperatures $T_{1}$ and $T_{2}$ of each couple $(b, g, c)$ in the RTG (from the preceding iteration of the analysis, or from initial guesses for the first iteration).

- Then, for each couple in the RTG, the code computes the temperature-averaged properties $k$ and $\rho$, the opencircuit voltage $V_{0}$, and the thermal conductance $K$ and electrical resistance $R$ :

$\tilde{k}_{n}(b, g, c)=\int_{T_{1(b, c)}}^{T_{2(b, c)}} k_{n}(T) d T /\left(T_{2}(b, g, c) \cdot T_{l}(b, g, c)\right)$

$\bar{k}_{p}(b, g, c)=\int_{T_{(b, c)}}^{T_{2(b, c)}} k_{p}(T) d T /\left[T_{z}(b, g, c) \cdot T_{1}(b, g, c)\right]$

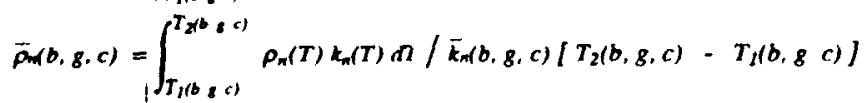

$\bar{\rho}_{p}(b, g, c)=\int_{T_{1(b, g)}}^{T_{2(b, c)}} \rho_{p}(T) k_{p}(T) d T / \vec{k}_{p}(b \& c)\left(T_{2}(b \&, c)-T_{l}(b, g c)\right]$

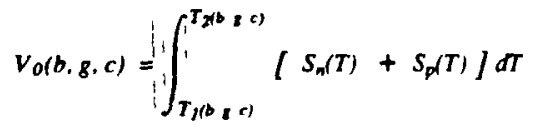

$K(b, g, c)=\left[\bar{k}_{n}(b, g, c) A_{n}+\tilde{k}_{p}(b g, c) A_{p}\right] / L$

$R(b, g, c)=\left[\bar{\rho}_{n}(b, g, c) / A_{n}+\bar{\rho}_{p}(b, g, c) / A_{p}\right] L$

In applying the above equations, the resistivity $\rho$ was increased by $6 \%$ to account for contact resistances and electrode losses, and all the thermoelectrlc properties $(S, p, k)$ were adjusted to account for degradation due to dopant precipitation at operating temperature. 


\section{Electrical Analysis}

The output voltage $V(b, g, c)$ of each couple equals its open-circuit voltage $V_{0}(b, g, c)$ less its intemal voltage drop $\mathbf{I}(\mathbf{b}, \mathbf{g}, \mathbf{c}) \mathbf{R}(\mathbf{b}, \mathbf{g}, \mathbf{c})$. Since the couples in each group $\mathbf{b}, \mathbf{g}$ are connected in parallel, they must have the same output voltage,

$$
V(b, g)=V_{0}(b, g, c) \cdot I(b, g, c) R(b, g, c) .
$$

Therefore, the current through couple b,g,c is:

$$
I(b, g, c)=\left[V_{0}(b, g, c)-V(b, g)\right] / R(b, g, c)
$$

The sum of each group's C couple currents equals the group current $\mathbf{l}(\mathbf{b}, \mathbf{g})$. Since all groups in each branch b are connected in series, this must equal the branch current,

$$
l(b)=\sum_{c=1}^{C} R^{-1}(b, g, c) V_{0}(b, g, c)-V(b, g) \sum_{c=1}^{C} R^{-l}(b, g, c)
$$

Therefore, the voltage produced by group $b, g$ is

$$
V(b, g)=\left[\sum_{c=1}^{C} R^{-l}(b, g, c) V_{D}(b, g, c)-I(b)\right]\left[\sum_{c=1}^{C} R^{-1}(b, g, c)\right]^{-I}
$$

The sum of the $\mathbf{G}$ group voltages in each branch equals the branch voltage $\mathbf{V}(\mathbf{b})$. Since the $B$ branches are connected in parallel, this also equals the output voltage of the RTG:

$$
V_{R T G}=\sum_{g=1}^{G}\left[\sum_{c=1}^{C} R^{-I}(b, g, c) V_{O}(b, g, c)\right]\left[\sum_{c=1}^{C} R^{-1}(b, g, c)\right]^{-1}-I(b) \sum_{g=1}^{G}\left[\sum_{c=1}^{C} R^{-1}(b, g, c)\right]^{-1} \text {. }
$$

Solving for the branch current I(b) for each branch and summing the currents for the B parallel branches gives the RTG output current:

$$
I_{R T G}=\sum_{b=1}^{B} \frac{\sum_{g=1}^{G}\left[\sum_{c=1}^{C} R^{-1}(b, g, c) V_{O}(b, g, c)\right]\left[\sum_{i=1}^{C} R^{-1}(b, g, c)\right]^{-1}-V_{R T G}}{\sum_{g=1}^{G}\left[\sum_{c=1}^{C} R^{-1}(b, g, c)\right]^{-1}} .
$$

\section{Computing the inputs for the Next Iteration}

For the prescribed RTG output voltage $\mathbf{V}_{\mathbf{R T G}}$ the code computes the branch current $\mathbf{I ( b )}$ for each of the $B$ branches,

$$
I(b)=\frac{\sum_{g=1}^{G}\left[\sum_{c=1}^{C} R^{-1}(b, g, c) V_{O}(b, g, c)\right]\left[\sum_{i=1}^{C} R^{-1}(b, g, c)\right]^{-1}-V_{R T G}}{\sum_{g=1}^{G}\left[\sum_{c=1}^{C} R^{-1}(b, g, c)\right]^{-1}},
$$

the group voltage $V(b, g)$ for each of the $G$ groups in each branch,

$$
V(b, g)=\left[\sum_{c=1}^{c} R^{-1}(b, g, c) V_{0}(b, g, c)-I(b)\right]\left[\sum_{c=1}^{c} R^{-1}(b, g, c)\right]^{-1}
$$

and the current $l(b, g, c)$ for each couple in the RTG,

$$
l(b, g, c)=\left[V_{0}(b, g, c)-V(b, g)\right] / R(b, g, c)
$$


11

The individual couple currents are then used to compute the hot-end heat input rate $Q_{H}(b, g, c)$ and the cold-end heat rejection rate $Q_{c}(b, g, c)$ for each couple in the RTG. Each of these rates consists of four terms: normal heat conduction, the Peltier effect, Onmic heating, and the Thomson effect. Expressions for these terms are summarized in Figure 5.

Figure 5. Unicouple Energy Balance

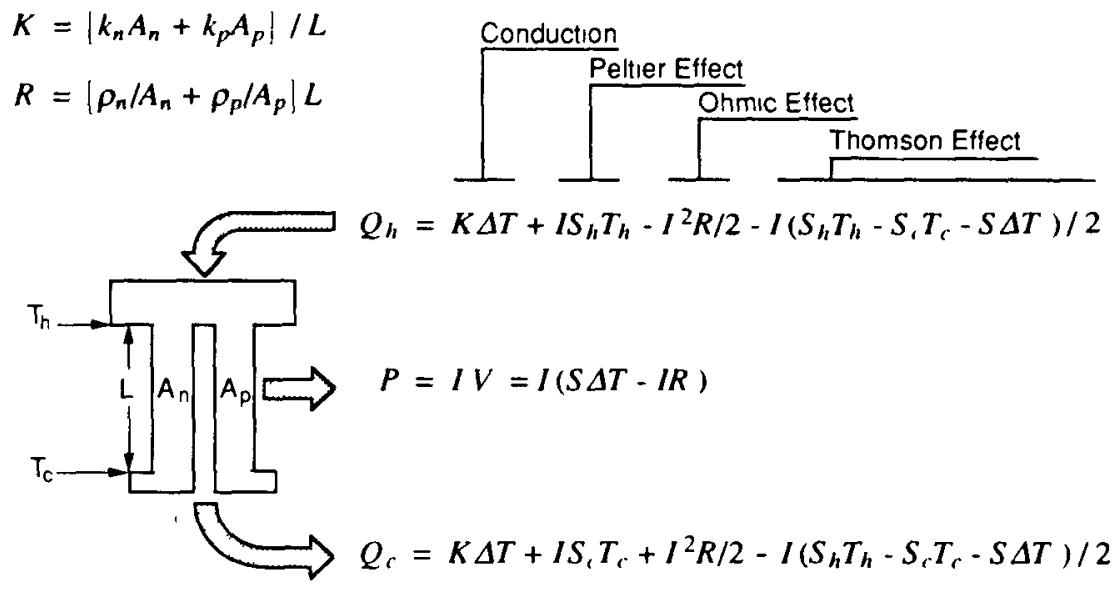

Three of the four terms comprising each heat flow rate are current-dependent. Therefore, the thermal and electrical analyses must be performed simultaneously. For each couple b,g,c in the RTG,

$$
\begin{aligned}
Q_{H}(b, g, c) & =K(b, g, c)\left[T_{2}(b, g, c)-T_{1}(b, g, c)\right]-I^{2}(b, g, c) R(b, g, c) / 2 \\
& +I(b, g, c)\left[S_{2}(b, g, c) T_{2}(b, g, c)+S_{I}(b, g, c) T_{I}(b, g, c)+V_{0}(b, g, c)\right] / 2, \\
Q_{C}(b, g, c) & =K(b, g, c)\left[T_{2}(b, g, c)-T_{I}(b, g, c)\right]+I^{2}(b, g, c) R(b, g, c) / 2 \\
& +l(b, g, c)\left[S_{2}(b, g, c) T_{2}(b, g, c)+S_{1}(b, g, c) T_{I}(b, g, c)-V_{o}(b, g, c)\right] / 2,
\end{aligned}
$$

where $S_{1}$ and $S_{2}$ denote the Seebeck coefficients at the cold and hot junction of the couple:

$$
\begin{aligned}
& S_{I}(b, g, c)=S_{n}\left[T_{I}(b, g, c)\right]+S_{p}\left[T_{I}(b, g, c)\right], \\
& S_{2}(b, g, c)=S_{n}\left[T_{2}(b, g, c)\right]+S_{p}\left[T_{2}(b, g, c)\right] .
\end{aligned}
$$

The code inserts these heat flow rates for each couple in the RTG into the detailed thermal analysis model, for the next iteration.

Note that $Q_{H}$ and $Q_{C}$ differ by the electrical power output of each couple:

$$
Q_{H}(b, g, c)-Q_{C}(b, g, c)=I(b, g, c)\left[V_{d}(b, g, c)-I(b, g, c) R(b, g, c)\right]
$$

This difference must be represented as an effective heat sink for eachlcouple in the thermal analysis model.

The computational sequence described above is repeated until the prescribed degree of convergence is achieved. 
The converged BOM solution for the baseline case, based on a thermal power of $\mathbf{4 2 3 0}$ watts from the 18-module heat source stack, is summarized in Tables 1 through 4. The tables display the results for the flattened-out cylindrical array of 576 thermocouples in one RTG. Table 1 shows the axial and circumferential variation of the RTG's cold-junction temperatures. As can be seen, these vary from $276^{\circ} \mathrm{C}$ for the least obstructed couple to $301^{\circ} \mathrm{C}$ for the most obstructed couple. The last column and last line of the table show the variation of the averaged cold-junction temperatures in the axial and circumferentlal directions, respectively. As can be seen, the average temperature is lowest near the ends of the RTG, particularly the upper (outboard) end, which is farthest from the spacecraft; and they are highest near the RTG's midplane. Circumferentially, the average temperature is lowest in Column 14, the RTG side furthest from its neighbor, and highest in Column 6 , the RTG side facing the other RTG.

In general, these results show that for the baseline design, the circumferential temperature variation is moderate, indicatıng that the two RTGs could be moved closer than 24 degrees to each other.

Table 2 similarly shows the axial and circumferential variations of the baseline RTG's hotjunction temperatures. As can be see, those temperatures vary only from $983^{\circ} \mathrm{C}$ to $1003^{\circ} \mathrm{C}$. The table also shows the axial variation of the heat source surface temperatures. As seen, these vary from $1015^{\circ} \mathrm{C}$ at the outboard end of the heat source stack to $1033^{\circ} \mathrm{C}$ near the middle of the stack.

As can be seen, the couples' averaged hotjunction temperatures vary by $19^{\circ} \mathrm{C}$ in the axial direction, and show essentially no variation in the circumferential direction.

Thus, the obstruction by the neighboring RTG affects only the cold-junction temperatures, and is not reflected in the hot-junction temperatures.

The consequent variation in the thermocouples' $\Delta T$ affects their electrical performance. The axial and circumferential variations of the couple voltages is displayed in Table 3, and those of the couple currents in Table 4. The sixteen columns in these tables represent eight column pairs of parallel couples. Table 3 shows that all parallel couples have the same output voltage; and Table 4 shows that all couple pairs in each branch have the same combined output current, as demanded by the RTG's series-parallel network. The respective output currents of Branches 1 and 2 were 4.68 amps and 4.70 amps, for a total RTG output of 9.38 amps. Each branch had an output voltage of 30.0 volts from its 144 series-connected couple groups. Thus, the RTG's power output is 281.5 watts. The average material efficiency of the couples is $7.34 \%$; the average couple efficiency (including the effect of contact resistances and electrode losses) is $7.00 \%$; and the total system efficiency (including the effect of heat losses through the thermal insulation and through the heat source support structure) is $6.65 \%$.
Table 1: COLD-JUNCTION TEMPERATORES $\left({ }^{\circ} \mathrm{C}\right)$

\begin{tabular}{c|rrrrrrrrrrrrrrrr|l} 
& 1 & 2 & 3 & 4 & 5 & 6 & 7 & 8 & 9 & 10 & 11 & 12 & 13 & 14 & 15 & 16 & AVG. \\
\hline 2 & 276 & 277 & 278 & 279 & 281 & 282 & 282 & 281 & 280 & 278 & 277 & 277 & 276 & 276 & 276 & 276 & 278 \\
4 & 278 & 279 & 280 & 281 & 283 & 284 & 284 & 283 & 282 & 280 & 279 & 278 & 278 & 278 & 277 & 277 & 280 \\
6 & 280 & 281 & 282 & 284 & 286 & 286 & 286 & 286 & 284 & 282 & 281 & 280 & 280 & 279 & 279 & 279 & 282 \\
8 & 281 & 282 & 284 & 286 & 288 & 289 & 289 & 288 & 286 & 284 & 283 & 282 & 281 & 281 & 281 & 281 & 284 \\
10 & 283 & 284 & 286 & 288 & 290 & 291 & 291 & 290 & 288 & 286 & 284 & 283 & 283 & 282 & 282 & 282 & 286 \\
12 & 284 & 285 & 287 & 289 & 292 & 293 & 293 & 292 & 290 & 287 & 286 & 284 & 284 & 284 & 283 & 283 & 287 \\
14 & 285 & 287 & 289 & 291 & 294 & 295 & 295 & 294 & 291 & 289 & 287 & 286 & 285 & 284 & 284 & 285 & 289 \\
16 & 286 & 288 & 290 & 293 & 295 & 297 & 297 & 296 & 293 & 290 & 288 & 287 & 286 & 285 & 286 & 286 & 290 \\
18 & 287 & 289 & 293 & 294 & 297 & 298 & 300 & 298 & 294 & 291 & 290 & 288 & 286 & 286 & 288 & 287 & 292 \\
20 & 288 & 290 & 293 & 295 & 298 & 299 & 301 & 299 & 295 & 292 & 291 & 288 & 287 & 287 & 288 & 287 & 292 \\
22 & 288 & 289 & 292 & 295 & 299 & 300 & 300 & 299 & 295 & 292 & 289 & 288 & 286 & 286 & 286 & 287 & 292 \\
24 & 287 & 289 & 292 & 295 & 299 & 300 & 300 & 299 & 295 & 291 & 289 & 287 & 286 & 286 & 286 & 286 & 292 \\
26 & 287 & 289 & 291 & 295 & 299 & 300 & 300 & 298 & 295 & 291 & 288 & 287 & 285 & 285 & 285 & 286 & 291 \\
28 & 286 & 288 & 291 & 294 & 298 & 299 & 299 & 298 & 294 & 290 & 288 & 286 & 285 & 284 & 284 & 285 & 291 \\
30 & 285 & 287 & 290 & 293 & 297 & 298 & 298 & 297 & 293 & 289 & 287 & 285 & 284 & 283 & 283 & 284 & 290 \\
32 & 284 & 286 & 288 & 292 & 296 & 297 & 297 & 296 & 292 & 288 & 285 & 284 & 282 & 282 & 282 & 282 & 288 \\
34 & 282 & 284 & 287 & 290 & 294 & 295 & 295 & 294 & 290 & 286 & 284 & 282 & 281 & 280 & 280 & 281 & 287 \\
36 & 280 & 282 & 285 & 288 & 292 & 293 & 293 & 292 & 288 & 285 & 282 & 281 & 279 & 279 & 279 & 279 & 285 \\
\hline
\end{tabular}

AVG. \begin{tabular}{llllllllllllllll|l}
284 & 285 & 288 & 290 & 293 & 294 & 294 & 293 & 290 & 287 & 285 & 284 & 283 & 283 & 283 & 283 & 288
\end{tabular}

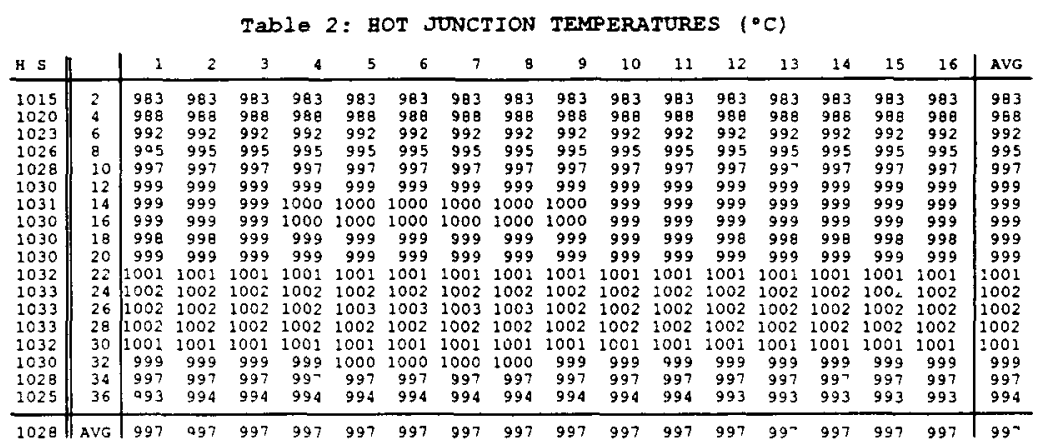

Table 3: COUPIE VOLTAGE (mV)

\begin{tabular}{c|ccccccccccccc|cc|ccc|cc|c} 
& 1 & 2 & 3 & 4 & 5 & 6 & 7 & 8 & 9 & 10 & 11 & 12 & 13 & 14 & 15 & 16 & AVG \\
\hline 2 & 204 & 204 & 206 & 206 & 206 & 206 & 206 & 206 & 205 & 205 & 204 & 204 & 203 & 203 & 203 & 203 & 205 \\
4 & 207 & 207 & 208 & 208 & 208 & 208 & 208 & 208 & 208 & 208 & 206 & 206 & 205 & 205 & 205 & 205 & 207 \\
6 & 208 & 208 & 209 & 209 & 210 & 210 & 210 & 210 & 209 & 209 & 208 & 208 & 206 & 206 & 206 & 206 & 208 \\
8 & 209 & 209 & 210 & 210 & 211 & 211 & 211 & 211 & 210 & 210 & 209 & 209 & 207 & 207 & 207 & 207 & 209 \\
10 & 209 & 209 & 211 & 211 & 212 & 212 & 211 & 211 & 211 & 211 & 209 & 209 & 207 & 207 & 208 & 208 & 210 \\
12 & 210 & 210 & 211 & 211 & 212 & 212 & 212 & 212 & 211 & 211 & 209 & 209 & 207 & 207 & 207 & 207 & 210 \\
14 & 209 & 209 & 211 & 211 & 212 & 212 & 212 & 212 & 211 & 211 & 209 & 209 & 207 & 207 & 207 & 207 & 210 \\
16 & 208 & 208 & 210 & 210 & 211 & 211 & 211 & 211 & 210 & 210 & 208 & 208 & 206 & 206 & 206 & 206 & 209 \\
18 & 207 & 207 & 209 & 209 & 210 & 210 & 210 & 210 & 209 & 209 & 207 & 207 & 205 & 205 & 204 & 204 & 208 \\
20 & 206 & 206 & 208 & 208 & 209 & 209 & 209 & 209 & 208 & 208 & 206 & 206 & 203 & 203 & 203 & 203 & 207 \\
22 & 207 & 207 & 210 & 210 & 211 & 211 & 211 & 211 & 210 & 210 & 207 & 207 & 204 & 204 & 204 & 204 & 208 \\
24 & 208 & 208 & 211 & 211 & 212 & 212 & 212 & 212 & 211 & 211 & 208 & 208 & 205 & 205 & 205 & 205 & 209 \\
26 & 208 & 208 & 211 & 211 & 212 & 212 & 212 & 212 & 211 & 211 & 208 & 208 & 205 & 205 & 205 & 205 & 209 \\
28 & 208 & 208 & 211 & 211 & 212 & 212 & 212 & 212 & 211 & 211 & 209 & 209 & 205 & 205 & 205 & 205 & 209 \\
30 & 208 & 208 & 211 & 211 & 212 & 212 & 212 & 212 & 211 & 211 & 208 & 208 & 205 & 205 & 205 & 205 & 209 \\
32 & 208 & 208 & 211 & 211 & 212 & 212 & 212 & 212 & 211 & 211 & 208 & 208 & 205 & 205 & 205 & 205 & 209 \\
34 & 207 & 207 & 210 & 210 & 211 & 211 & 211 & 211 & 210 & 210 & 207 & 207 & 204 & 204 & 204 & 204 & 208 \\
36 & 206 & 206 & 209 & 209 & 210 & 210 & 210 & 210 & 209 & 209 & 206 & 206 & 203 & 203 & 203 & 203 & 207 \\
\hline AVG & 208 & 208 & 210 & 210 & 211 & 2031 & 211 & 203 & 211 & 210 & 210 & 208 & 208 & 205 & 205 & 205 & 205 & 208
\end{tabular}

Table 4: COUPLE CURRENT (amp)

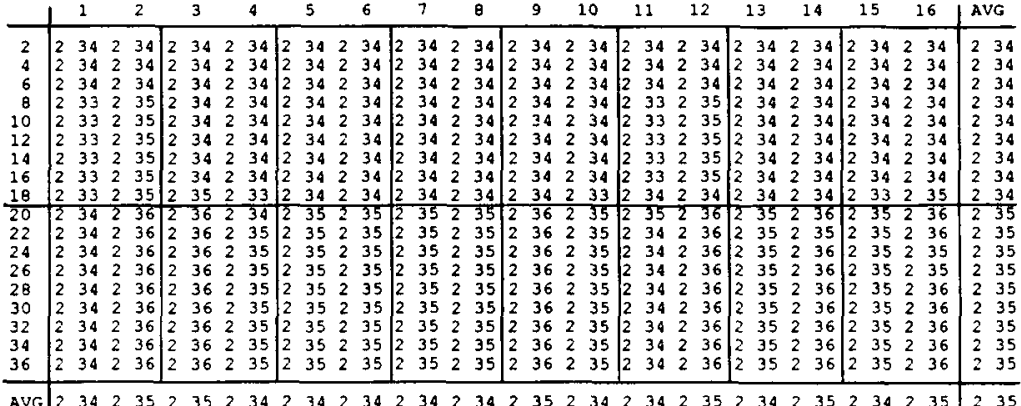




\section{ILLUSTRATIVE APPLICATION}

As mentioned in the Introduction, the methodology deveioped for the analysis of obstructed ATGs was applied to the specific case of the RTGs for the CRAF and Cassini missions, which will each use two GPHS-RTGs aboard a Mariner Mark-II spacecraft. Various RTG locations and orientations are under consideration by JPL's spacecraft designers. The one that is currently baselined is depicted in Figure 6. The two RTGs are cantilevered radially from the cylindrical spacecraft hub, with a separation angle of 24 degrees between their axes. As can be seen, each RTG is obstructed by its partner and by nearby spacecraft components.

Figure 6. CRAF-CASSINI Spacecraft, Baseline Design

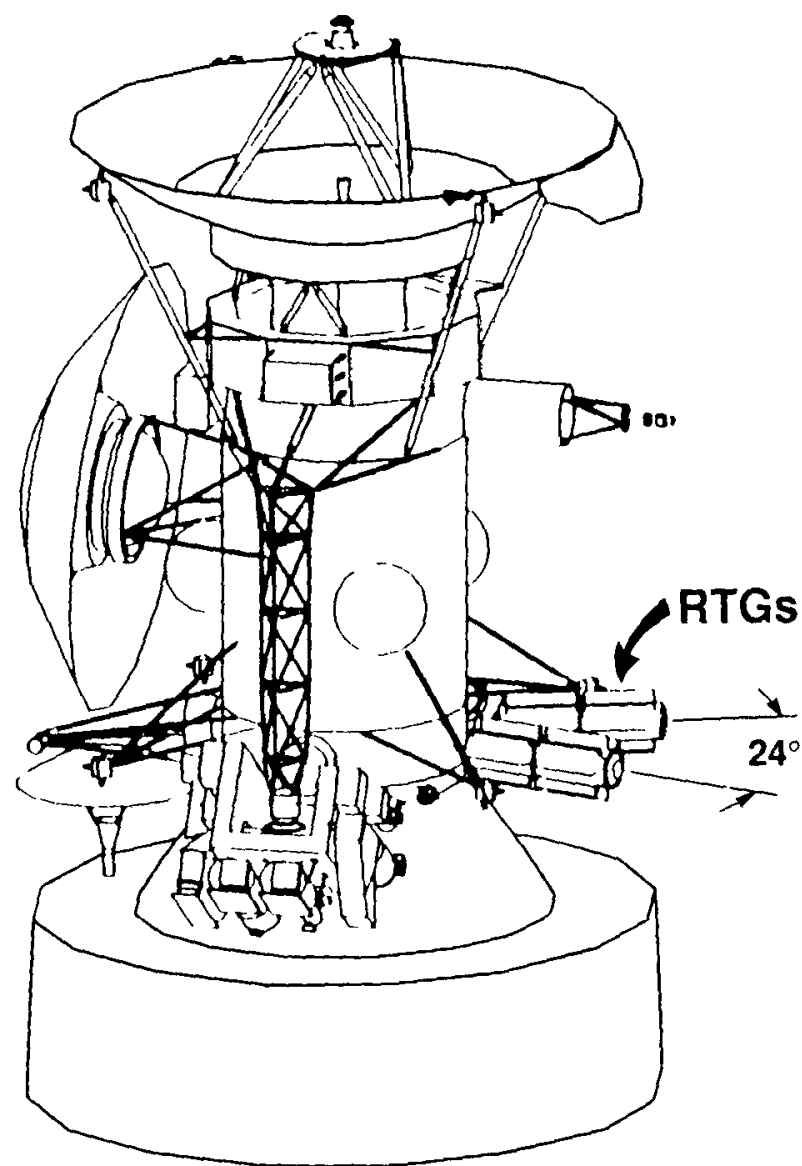

An alternative RTG orientation which JPL asked Fairchild to analyze is depicted in Figure 7. It is quite similar to the baseline configuration, except that the two RTGs are tilted down from the horizontal plane. Various separation angles and tilt angles will be analyzed. Clearly, either configuration can result in significant axial and circumferential temperature variations. Only the results for the baseline configuration will be presented in detail.

The analysis of the CRAF/Cassini RTGs started with a very detailed radiation interchange analysis model, representing the housing and fins of both RTGs and all
Figure 7. Alternate RTG Orientation

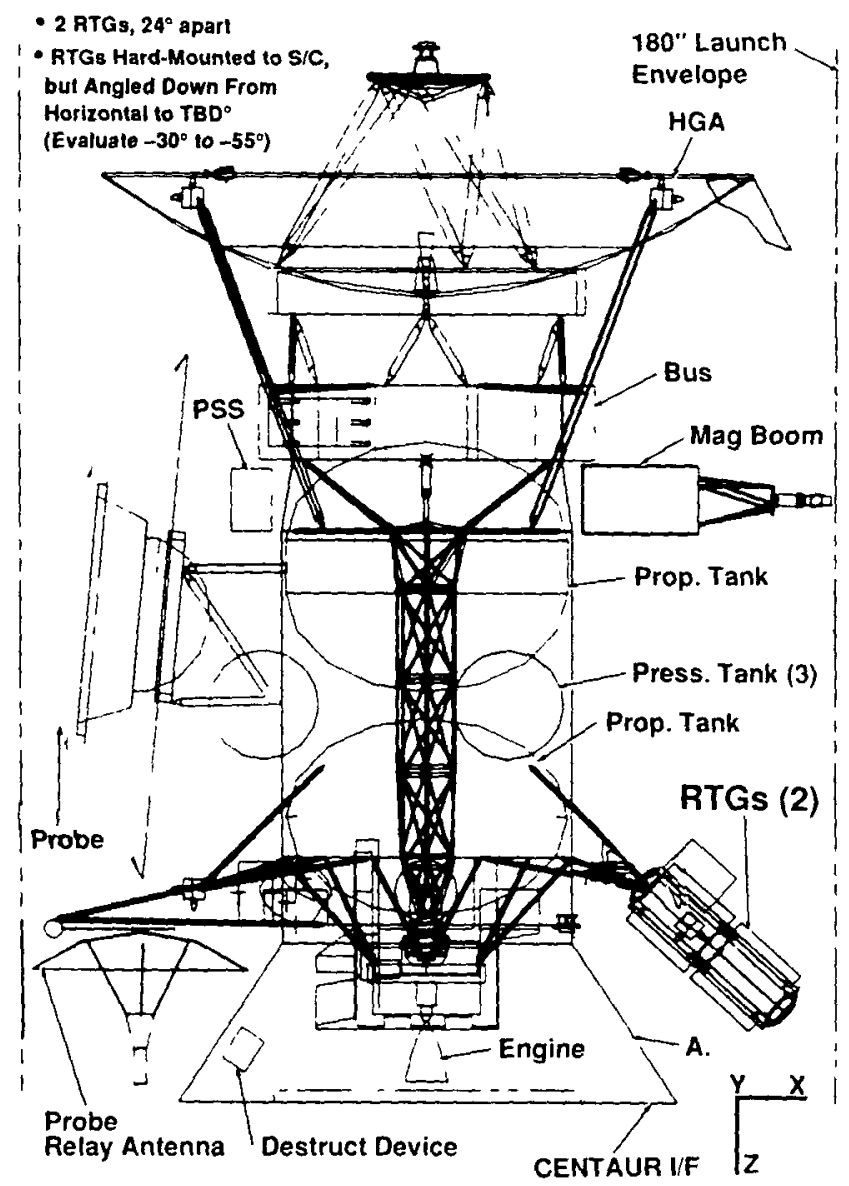

spacecraft surfaces that significantly obstruct their view of space. The model consisted of a total of 1912 surface nodes. The ITAS (Integrated Thermal Analysis System) code [7], which accounts for the effect of mutual reflections, was used to compute over 102,000 radiation interchange factors between all surface nodes that are within each other's view.

The computed radiation interchange factors were then inserted into a detalled thermal and electrical analysis model consisting of - 2900 node points. The thermal power of the 18 stacked-up heat source modules (initially 250 watts) was adjusted to account for fuel decay during the anticipated interval between the fuel encapsulation and the time of interest, usually the beginning-of-mission (BOM) or end-of-mission (EOM). The coupled thermal and electrical analysis was carried out by means of a Fairchildgenerated computer code. The code was based on the SINDA thermal analysis program [8], modified to incorporate the current-dependent Peltier, Ohmic, and Thomson effects on thermocouple conductance and to represent the electrical power generation in each couple as an effective heat sink. These corrections were computed and applied in each iteration. After the solution had homed in to prescribed convergence criteria, it was used to calculate the RTG's electrical output and efficiency. 
In addition to the baseline case with a thermal power of 235 watts in each of the 18 heat source modules, identical analyses of obstructed RTGs with thermal power of $230,240,245$, and 250 watts per heat source module were carried out. The final results for the five cases are displayed by the lower curve of Figure 8 . The curve shows the effect of the thermal power and the corresponding maximum hot-junction temperature on RTG power output.

To assess the effect of the obstruction by the neighboring RTG on performance, similar detalled analyses were performed for an identical RTG without obstructions. For that case, the RTG had axial varlations but no circumferential variations. These cases, with thermal powers of 240,245 , and 250 watts per heat source module, were analyzed. The final results for the unobstructed RTGs are shown by the upper curve of Figure 8 .

Figure 8. Effect of Thermal Power and Maximum Hot-Junction Temperature on Power Output of Baseline RTG

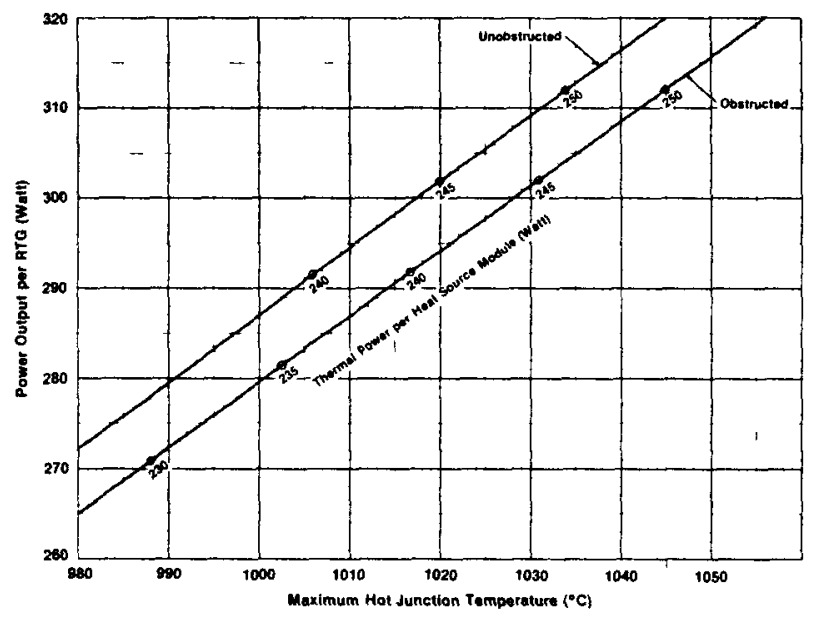

Figure 8 shows that for the same thermal power, the obstructed and unobstructed RTGs yield roughly the same power output. But that is a misleading conclusion, because the obstructed case ylelds significantly higher maximum hot-function temperatures. The allowable service life of a thermoelectric couple is a rather sensitive function of its hotjunction temperature. Moreover, the higher the hot-junction temperatures, the higher the temperature of the iridium clads that encapsulate the radioisotope fuel. Excessively high clad temperatures can lead to excessive grain growth during high-temperature operation. This can embrittle the iridium and reduce the safety margin in case of accidental reentry and earth impact.

For these reasons, the maximum hot-junction temperature for long-term operation is generally llmited to $1000^{\circ} \mathrm{C}$. Comparison between obstructed and unobstructed RTGs should be made for the same maximum hot-junction temperature rather than the same thermal power. Figure 8 shows that for a maximum hot-junction temperature of $1000^{\circ} \mathrm{C}$, the power output of the obstructed RTG is about 7 watts (or $2.4 \%$ ) lower than that of the unobstructed RTG. This is a fairly modest penalty, which confirms the previous conclusion that it may be practical to move the two RTGs closer together, if desired. To permit moving them together without interference between their fins, one of the two RTGs is rotated 22.5 degrees about its axis. That option is now undergoing analysis, to determine the trade-off between RTG proximity and pertormance. The trade-off results will be used to guide the spacecraft design.

The results presented above were for a spacecraft emissivity of 0.85 . Additional analyses were performed to assess the effect of spacecraft emissivity on the RTG's temperatures and performance. The results indicated that the RTG's power output is virtually independent of the spacecraft's reflectivity.

A special analysis was carried out for the case of the CRAF spacecraft, which is planned to have one freshly fueled RTG and one old RTG that had been an unneeded spare for the Galileo mission. Since the tuel of the old RTG had been encapsulated in 1982, about eleven years before that of the new RTG, it will have undergone much greater isotope decay by the time of launch. Thus, the two neighboring RTGs will start the mission with substantially different thermal powers and temperatures. A detailed analysis was carried out with the two RTGs using heat source modules of 245 and 227 watts, respectively. The results for each RTG fell almost exactly on the lower curve of Figure 8. Thus, the effect of an obstructive RTG on its neighbor's performance is insensitive to the obstructor's temperature.

In addition to the baseline configuration, a detailed analysis of the tilted-RTG alternative depicted in Figure 7 was carried out. The results were almost identical to the horizontal-RTG baseline case. Under these circumstances, there is little incentive for going to the tilted-RTG option, especially since that option may lead to undesirable effects of the rocket exhaust plume on the inboard side of the RTG.

As seen, the computer code described in this paper has proved to be a versatile and useful tool for the detailed coupled thermal, thermoelectric, and electrical analysis of unsymmetrical RTGs with complex couple networks.

\section{REFERENCES}

11] A Schock, "Modular Isotopic Thermoelectric Generator " Proceedings of the 1981 Intersociety Energy Conversion Engineering Conference, Volume 1, peges $327-342$

[2] A Schock, T Or, and E Skrabek, "Thermal and Electrical Analysis of Mars Rover RTGs " Proceedings of the 24th Intersociety Energy Conversion Engineening Conference, 611 August, 1989

[3] A Schock, "Mars Rover RTa Study " 40th Congress of the International Astronautical Federation, held in Malaga, Spain, 7-13 October 1989

[4] A Schock, CT Or, and E A Skrabek Thermal and Electrical Analysis of Mars Rover RTG, and Performance Comparison of Alternative Design Options " Trans of the 7th Symposium on Space Nuciear Power Systems Abuquerque, NM, January 1990

[5] R D Cockfield, R F Hartman, C D Kelly, "RTG Power Sources for International Solar Polar Mission " Proceedings of the 15th Intersociety Energy Conversion Engineering Conference, held in Seattle WA, 1980, $\$ 809204$

[6] R D Cockfield "Qualification of GPHS RTG for Galleo and Ulysses" Proceedings of the 21 st Intersociety Energy Conversion Engineering Conferenco, held in San Diego, CA, 1986

[7] ITAS Integrated Thermal Analysis Systems Analytix Corporation Timonium, Maryland, 1990

[8] Gaskı J (1986) SINDA (Syslem Improved Numerical Differencing Analyzer) version 123 from Network Analysis Associate Fountain Valley Calitornta 